\title{
Diffusion equation and spin drag in spin-polarized transport
}

\author{
Flensberg, Karsten; Jensen, Thomas Stibius; Mortensen, Asger
}

Published in:

Physical Review B Condensed Matter

Link to article, DOI:

10.1103/PhysRevB.64.245308

Publication date:

2001

Document Version

Publisher's PDF, also known as Version of record

Link back to DTU Orbit

Citation (APA):

Flensberg, K., Jensen, T. S., \& Mortensen, A. (2001). Diffusion equation and spin drag in spin-polarized

transport. Physical Review B Condensed Matter, 64(24), 245308. https://doi.org/10.1103/PhysRevB.64.245308

\section{General rights}

Copyright and moral rights for the publications made accessible in the public portal are retained by the authors and/or other copyright owners and it is a condition of accessing publications that users recognise and abide by the legal requirements associated with these rights.

- Users may download and print one copy of any publication from the public portal for the purpose of private study or research.

- You may not further distribute the material or use it for any profit-making activity or commercial gain

- You may freely distribute the URL identifying the publication in the public portal

If you believe that this document breaches copyright please contact us providing details, and we will remove access to the work immediately and investigate your claim 


\title{
Diffusion equation and spin drag in spin-polarized transport
}

\author{
Karsten Flensberg, ${ }^{1}$ Thomas Stibius Jensen, ${ }^{1}$ and Niels Asger Mortensen ${ }^{1,2}$ \\ ${ }^{1}$ Orsted Laboratory, Niels Bohr Institute fAPG, Universitetsparken 5, 2100 Copenhagen, Denmark \\ ${ }^{2}$ Mikroelektronik Centret, Technical University of Denmark, 2800 Lyngby, Denmark
}

(Received 6 July 2001; published 29 November 2001)

\begin{abstract}
We study the role of electron-electron interactions for spin-polarized transport using the Boltzmann equation, and derive a set of coupled transport equations. For spin-polarized transport the electron-electron interactions are important, because they tend to equilibrate the momentum of the two-spin species. This "spin drag" effect enhances the resistivity of the system. The enhancement is stronger the lower the dimension is, and should be measurable in, for example, a two-dimensional electron gas with ferromagnetic contacts. We also include spin-flip scattering, which has two effects: it equilibrates the spin density imbalance and, provided it has a non-s-wave component, also a current imbalance.
\end{abstract}

DOI: 10.1103/PhysRevB.64.245308

PACS number(s): 72.25.Ba, 72.25.Rb, 72.25.Dc

\section{INTRODUCTION}

Recent advances in the fabrication of ferromagneticsemiconductor heterostructures ${ }^{1}$ and the observation of spin injection into semiconductors ${ }^{2}$ have lead to interest in the transport properties of spin-polarized systems. There has been considerable work done in the field of metallic magnetic multilayers, which has been analyzed in terms of transport equations with spin dependent distribution functions. ${ }^{3,4}$ These works based their analysis on diffusion transport equations. The justification of using these equations was given by Valet and Fert, ${ }^{5}$ who derived a spin diffusion equation from the Boltzmann equation in the limit where the spin scattering length is much longer than the momentum relaxation length. Recently, the transport equations were utilized to analyze the feasibility of spin injection into semiconductors, with the result that the crucial parameter is the conductivity mismatch between the semiconductor and the ferromagnet, ${ }^{6}$ and to study spin-polarized transport theoretically in inhomogeneous doped semiconductors. ${ }^{7}$

None of these approaches took electron-electron $(e-e)$ scattering into account. Clearly $e-e$ interactions play a different role than in usual spin degenerate transport, where the $e-e$ interaction does not provide a mechanism for momentum relaxation and hence has only indirect consequences for transport coefficients. In spin-polarized transport the two spin species have different drift velocities, and $e-e$ interactions are instrumental in equilibrating this difference. This leads to a spin drag effect where the spins carrying the larger current will drag along the spins carrying the smaller current. This drag effect was recently considered by D'Amico and Vignale $^{8,9}$ in three dimensions using linear response theory. They found that the spin drag resistivity was appreciable, and at elevated temperatures can be a fraction of the usual resistivity of the metal. In two dimensions the effect of $e-e$ interactions on spin diffusion was considered theoretically by Takahashi et al. ${ }^{10,11}$ Using a quantum kinetic equation approach, previously utilized in ${ }^{3} \mathrm{He}-{ }^{4} \mathrm{He}$ solutions, ${ }^{12}$ they studied the spin diffusion coefficients in two-dimensional electron gases. In order to study this spin diffusion they used variational functions, but did not include spin-relaxation scattering.
In this paper, we use the Boltzmann equation to study spin-dependent transport and spin diffusion. We restrict ourselves to the study of collinear magnetization, and our goal is to derive a set of transport equation in the semiclassical limit. For this purpose the Boltzmann equation is adequate. For the noncolinear case, where phenomena such as damped transverse spin modes can occur, one must go beyond the present approach; see, e.g., Refs. 10 and 11 and references therein. We include impurity scattering, both spin independent and spin flip scattering, as well as $e$-e scattering. We show that a shifted Fermi-Dirac (SFD) distribution, is a valid solution at low temperatures $T \ll T_{F}$, and without spin-flip scattering. This is also the case for weak $e-e$ scattering, where the problem in absence of spin-flip reduces to the ordinary Coulomb drag situation. ${ }^{13,14}$

We then go on to discuss the general case at higher temperatures, general interaction strength and finite intrinsic spin-flip scattering. Using a SFD ansatz, for an isotropic system we find the following macroscopic transport equations:

$$
\begin{gathered}
\nabla \cdot \mathbf{J}_{s}=\left(-\frac{e}{\tau_{\mathrm{sf}}^{0}} \frac{\partial n_{s}^{0}}{\partial \mu}\right)\left(\bar{\mu}_{s}-\bar{\mu}_{-s}\right), \\
\nabla \bar{\mu}_{s}=\frac{e}{\sigma_{s}} \mathbf{J}_{s}+\left(\frac{e}{\sigma_{D} \alpha_{s}}+\frac{e}{\sigma_{\mathrm{sf}, \mathrm{s}}}\right)\left(\mathbf{J}_{s}-\alpha_{s} \mathbf{J}_{-s}\right) .
\end{gathered}
$$

Here $J_{s}$ is the current carried by electrons with spin $s, \bar{\mu}_{s}$ is the local spin-dependent electrochemical potential, $\sigma_{s}$ is the conductivity of the spin $s$ electron gas, $\tau_{\mathrm{sf}}^{0}$ is a spin lifetime due to intrinsic spin-flip processes, $\sigma_{\text {sf,s }}$ is a spin current conversion conductivity arising from the angle dependence of the spin-flip scattering, and $\alpha_{s}=n_{s}^{0} / n_{-s}^{0}$ is the relative spin density, see Eqs. (35) and (39) for definitions. Finally $\sigma_{D}$ is the spin drag conductivity, given by

$$
\begin{aligned}
\left(\sigma_{D}\right)^{-1}= & \frac{\hbar^{2}}{d e^{2} n_{s} n_{-s}} \int \frac{d \mathbf{q}}{(2 \pi)^{d}} \int_{0}^{\infty} d \omega q^{2}|e \phi(q)|^{2} \\
& \times \frac{\operatorname{Im} \chi_{s}(q, \omega) \operatorname{Im} \chi_{-s}(q, \omega)}{k_{B} T \sinh ^{2}\left(\hbar \omega / 2 k_{B} T\right)},
\end{aligned}
$$


where $\operatorname{Im} \chi_{s}$ is the polarization function:

$$
\begin{aligned}
\operatorname{Im} \chi_{s}(q, \omega)= & \pi \int \frac{d \mathbf{k}}{(2 \pi)^{d}}\left[f_{s}^{0}(|\mathbf{k}+\mathbf{q}|)\right. \\
& \left.-f_{s}^{0}(k)\right] \delta\left(\varepsilon|\mathbf{k}+\mathbf{q}| s-\varepsilon_{k s}-\hbar \omega\right) .
\end{aligned}
$$

Formula (2) is well known from Coulomb drag. ${ }^{13}$ At low temperatures it is $\left(\sigma_{D}\right)^{-1} \propto T^{2}$ in two and three dimensions, while in one dimension it is proportional to $T$; see, e.g., Ref. 15.

The first transport equation [Eq. (1a)] is the continuity equation, which expresses the conservation of current in the presence of spin-flip processes. The second equation [Eq. (1b)] is a generalized Ohm's law. The first term on the righthand side is Ohm's law, while the second term shows that a momentum imbalance between the two spin directions gives rise to an additional resistance if there is a mechanism for conversion of the spin current. There are two such processes possible. This first one is the spin drag effect mentioned above, where $e-e$ scattering makes a transfer of momentum possible. The second one is due to the elastic spin-flip scattering on, for example, magnetic impurities, which can convert a current with one spin polarization to a current of the opposite polarization, if the spin-flip matrix element has an angular dependence. For example if the spin-flip predominant scatters forward, this means that spin-flip scattering is accompanied by a transfer of momentum. In contrast if the spin-flip scattering, is purely $s$-wave scattering the momentum transfer between the spin channels is on average equal to zero. This can be seen mathematically from the expression for $\tau_{\mathrm{sf}}$ in Eq. (35c). The derivation of these two terms is the main result of the present paper.

Two consequences of the spin current relaxation terms can immediately be read off. First, they give rise to an increased resistivity in the case where the current is spin polarized. For example, taking $J_{\downarrow}=0$, the effective resistivity for electrons with spin $\uparrow$ becomes $\left(1 / \sigma_{\uparrow}+1 / \sigma_{D} \alpha_{s}\right.$ $\left.+1 / \sigma_{\mathrm{sf}, \mathrm{s}}\right)^{-1}$, and hence is an enhanced resistivity. Second, from Eqs. (1) we obtain a diffusion equation for the electrochemical potential difference

$$
\nabla^{2}\left(\bar{\mu}_{s}-\bar{\mu}_{-s}\right)=\frac{\bar{\mu}_{s}-\bar{\mu}_{-s}}{l_{\mathrm{sf}}^{2}},
$$

where

$$
\frac{1}{l_{\mathrm{sf}}^{2}}=\left[-\frac{e^{2}}{\tau_{\mathrm{sf}}^{0}} \frac{\partial n_{s}^{0}}{\partial \mu}\right] \sum_{s}\left[\frac{1}{\sigma_{s}}+\left(\frac{1}{\sigma_{\mathrm{sf}, \mathrm{s}}}+\frac{1}{\sigma_{D} \alpha_{s}}\right)\left(1+\alpha_{s}\right)\right] .
$$

This shows that the intrinsic spin relaxation length is decreased by the spin-drag- and angle-dependent spin-flip effects.

Similarly, we obtain that the following weighted sum of electro chemical potentials must vanish,

$$
\nabla^{2}\left(c_{-s} \bar{\mu}_{s}+c_{s} \bar{\mu}_{-s}\right)=0,
$$

where

$$
c_{s}=\frac{\partial n_{s}^{0}}{\partial \mu} \frac{1}{\sigma_{s}}+\left(\frac{1}{\sigma_{\mathrm{sf}, s}}+\frac{1}{\sigma_{D} \alpha_{s}}\right)\left(\frac{\partial n_{s}^{0}}{\partial \mu}+\alpha_{s} \frac{\partial n_{-s}^{0}}{\partial \mu}\right)
$$

Below, we derive Eqs. (1a) and (1b) and estimate the spin drag contributions. For the two-dimensional case, we also perform the integration of Eq. (2) numerically.

\section{BOLTZMANN EQUATION FOR COLLINEAR SPIN TRANSPORT}

We base our analysis on the Boltzmann equation for transport through a system with lifted spin degeneracy. We take the current to run in the $x$ direction, and denote the nonequilibrium distribution function by $f_{s}(\mathbf{k})$ and the equilibrium Fermi-Dirac distribution function by $f_{0}$,

$$
f_{0}\left(\varepsilon_{k s}\right)=\frac{1}{e^{\beta\left(\varepsilon_{k s}-\mu_{0}\right)}+1},
$$

where $\mu_{0}$ is the chemical potential and $\beta$, as usual, the inverse temperature. The eigenenergies are denoted $\varepsilon_{k s}$, where $s$ is the spin quantum number and $\mathbf{k}$ the quantum number labeling the relevant states crossing the Fermi level. For simplicity, we assume a parabolic dispersion and write

$$
\varepsilon_{k s}=\frac{\hbar^{2} k^{2}}{2 m}+\varepsilon_{s}^{0},
$$

where $\varepsilon_{s}$ is the band offset which can be spin dependent if the material is ferromagnetic.

The linearized Boltzmann equation then reads

$$
v_{x}(\mathbf{k}) \frac{\partial f_{s}(\mathbf{k}, x)}{\partial x}-\frac{e E_{x}}{\hbar} \frac{\partial f_{0}\left(\varepsilon_{k s}\right)}{\partial k_{x}}=\left(\frac{\partial f_{s}(\mathbf{k}, x)}{\partial t}\right)_{\text {coll. }} .
$$

We take the collision integral to include elastic scattering and $e$-e scattering,

$$
\begin{aligned}
\left(\frac{\partial f_{s}(\mathbf{k})}{\partial t}\right)_{\text {coll. }}= & H_{0}\left[f_{s}\right](\mathbf{k})+H_{\mathrm{sf}}\left[f_{s}, f_{-s}\right](\mathbf{k})+H_{e-e}\left[f_{s}, f_{s}\right](\mathbf{k}) \\
& +H_{e-e}\left[f_{s}, f_{-s}\right](\mathbf{k}),
\end{aligned}
$$

where $H_{0}$ is the scattering from impurities (or quasielastic phonon scattering), giving rise to a momentum relaxation

$$
\begin{aligned}
H_{0}\left[f_{s}\right](\mathbf{k})= & -\int \frac{d \mathbf{k}^{\prime}}{(2 \pi)^{d}} W_{s}^{0}\left(\mathbf{k}, \mathbf{k}^{\prime}\right) \\
& \times\left[f_{s}(\mathbf{k})-f_{s}\left(\mathbf{k}^{\prime}\right)\right] \delta\left(\varepsilon_{k s}-\varepsilon_{k^{\prime} s}\right),
\end{aligned}
$$

and where $H_{s f}$ describes elastic scattering processes that flip the spin:

$$
\begin{aligned}
H_{\text {sf }}\left[f_{s}, f_{-s}\right](\mathbf{k})= & -\int \frac{d \mathbf{k}^{\prime}}{(2 \pi)^{d}} W_{s f}\left(\mathbf{k}, \mathbf{k}^{\prime}\right)\left[f_{s}(\mathbf{k})\right. \\
& \left.-f_{-s}\left(\mathbf{k}^{\prime}\right)\right] \delta\left(\varepsilon_{k s}-\varepsilon_{k^{\prime}-s}\right) .
\end{aligned}
$$

Finally, the $e-e$ scattering is after the linearization given by ${ }^{16}$ 


$$
\begin{aligned}
H_{e-e}\left[f_{s}, f_{s^{\prime}}\right] 7(\mathbf{k})= & -\frac{2 \pi}{\hbar k T} \int \frac{d \mathbf{k}^{\prime}}{(2 \pi)^{d}} \int \frac{d \mathbf{q}}{(2 \pi)^{d}} \\
& \times\left|U_{s s^{\prime}}\left(\mathbf{q}, \varepsilon_{\mathbf{k}}-\varepsilon_{\mathbf{k}+\mathbf{q}}\right)\right|^{2} \delta\left(\varepsilon_{\mathbf{k}}+\varepsilon_{\mathbf{k}^{\prime}}-\varepsilon_{\mathbf{k}+\mathbf{q}}\right. \\
& \left.-\varepsilon_{\mathbf{k}^{\prime}-\mathbf{q}}\right) f_{0}\left(\varepsilon_{k s}\right) f_{0}\left(\varepsilon_{k^{\prime} s^{\prime}}\right) \\
& \times\left[1-f_{0}\left(\varepsilon_{|\mathbf{k}+\mathbf{q}| s}\right)\right]\left[1-f_{0}\left(\varepsilon_{\left|\mathbf{k}^{\prime}-\mathbf{q}\right| s^{\prime}}\right)\right] \\
& \times\left[\Psi_{s}(\mathbf{k})+\Psi_{s^{\prime}}\left(\mathbf{k}^{\prime}\right)-\Psi_{s}(\mathbf{k}+\mathbf{q})\right. \\
& \left.-\Psi_{s^{\prime}}\left(\mathbf{k}^{\prime}-\mathbf{q}\right)\right],
\end{aligned}
$$

where the deviation from equilibrium is expressed in the function $\Psi$ through

$$
f_{s}(\mathbf{r}, \mathbf{k})=f_{s}^{0}(k)+\left(-\frac{\partial f_{0}\left(\varepsilon_{k s}\right)}{\partial \varepsilon_{k s}}\right) \Psi_{s}(\mathbf{r}, \mathbf{k}) .
$$

The interaction $U_{s s^{\prime}}$ is the Coulomb interaction between two electrons with spin $s$ and $s^{\prime}$. It can in principle depend on the relative direction of the spin if exchange is included. This set of integral equations cannot be solved in general, and one must either solve them numerically (for example as in Ref. 17), or proceed with approximate methods.

However, one simplification is possible from symmetry. Because of the cylindrical symmetry the functions $\Psi_{s}(\mathbf{k})$ only depend on the angle between $\mathbf{k}$ and the direction of the current, which we here choose to be in the $x$ direction. Denoting this angle by $\theta$, we have $\cos \theta=\mathbf{k} \cdot \hat{\mathbf{x}} / k$, and we can write

$$
\Psi_{s}(\mathbf{r}, \mathbf{k})=\Psi_{s}(x, k, \theta) .
$$

It is convenient to expand the distribution function in harmonics of the angle $\theta$ as

$$
\Psi_{s}(x, k, \theta)=\sum_{n=0}^{\infty} g_{s}^{(n)}(x, k) \cos n \theta,
$$

which we utilize in Sec. III.

\section{SPIN DRAG WITHOUT SPIN-FLIP PROCESSES FOR $T \ll T_{F}$}

In this section we study the Boltzmann equation in the presence of $e-e$ interaction, but in the absence of spin-flip processes, i.e., $H_{s f}=0$. Furthermore, because a lowtemperature expansion allows for a solution of the Boltzmann equation, we start by examining this limit, and later we discuss the validity of this solution even at elevated temperatures. It turns out that the solution in the low-temperature regime corresponds to a SFD distribution.

In the low-temperature limit, we see from Eq. (10) that the second term on the left-hand side (the driving term) restricts $\varepsilon_{\mathbf{k} s}$ to lie close to the Fermi level, such that the deviation $\Psi_{s}(\mathbf{k})$ needs only to be evaluated at $k_{F}$. This is therefore also true for the distribution function in the elastic collision term, $H_{0}$. Due to the Pauli principle, this will also be the case for the $\Psi^{\prime} \mathrm{s}$ in the $e$-e collision integrals, which is seen as follows. Using standard tricks (see, e.g., Ref. 13), we rewrite the $e$-e collision term as

$$
\begin{aligned}
H_{e-e}\left[f_{s}, f_{s^{\prime}}\right](\mathbf{k})= & -\frac{2 \pi}{\hbar} \int_{-\infty}^{\infty} \frac{d \omega}{2 \pi} \int \frac{d \mathbf{k}^{\prime}}{(2 \pi)^{d}} \int \frac{d \mathbf{q}}{(2 \pi)^{d}} \\
& \times \mid U_{s s^{\prime}}\left(\mathbf{q}, \varepsilon_{k}-\left.\varepsilon_{|\mathbf{k}+\mathbf{q}|)}\right|^{2}\right. \\
& \times \frac{1}{k T \sinh ^{2}\left(\hbar \omega / k_{B} T\right)} \operatorname{Im} \chi_{s}(\mathbf{k}, \mathbf{q} ; \omega) \\
& \times \operatorname{Im} \chi_{s^{\prime}}\left(\mathbf{k}^{\prime},-\mathbf{q} ; \omega\right)\left[\Psi_{s}(\mathbf{k})+\Psi_{s^{\prime}}\left(\mathbf{k}^{\prime}\right)\right. \\
& \left.-\Psi_{s}(\mathbf{k}+\mathbf{q})-\Psi_{s^{\prime}}\left(\mathbf{k}^{\prime}-\mathbf{q}\right)\right],
\end{aligned}
$$

where

$$
\begin{aligned}
\operatorname{Im} \chi_{s}(\mathbf{k}, \mathbf{q} ; \omega)= & \pi\left[f_{0}\left(\varepsilon_{|\mathbf{k}+\mathbf{q}| s}\right)-f_{0}\left(\varepsilon_{k s}\right)\right] \\
& \times \delta\left(\varepsilon_{|\mathbf{k}+\mathbf{q}| s}-\varepsilon_{k s}-\hbar \omega\right) .
\end{aligned}
$$

Now, at low temperatures the factor $1 / \sinh ^{2}$ restricts the $\omega$ integral to small $\omega$ of order $k T$, and hence $\varepsilon_{|\mathbf{k}+\mathbf{q}| s}$ in Eq. (19) deviates from $\varepsilon_{k s}$ by an amount of order $k T$, and we expand $\operatorname{Im} \chi_{s}$ as

$$
\operatorname{Im} \chi_{s}(\mathbf{k}, \mathbf{q} ; \omega) \approx \pi \hbar \omega\left(-\frac{\partial f_{0}\left(\varepsilon_{k s}\right)}{\partial \varepsilon_{k s}}\right) \delta\left(\varepsilon_{|\mathbf{k}+\mathbf{q}| s}-\varepsilon_{k s}-\hbar \omega\right) .
$$

From this we conclude that both $\varepsilon_{k s}$ and $\varepsilon_{k^{\prime} s^{\prime}}$ (and hence also $\varepsilon_{|\mathbf{k}+\mathbf{q}| s}$ and $\left.\varepsilon_{\left|\mathbf{k}^{\prime}-\mathbf{q}\right| s^{\prime}}\right)$ are within a shell of order $k_{B} T$ from the Fermi level. To leading order in $k T / \varepsilon_{F}$, we can therefore neglect the dependence on $k$ and keep only the angular dependence of $\Psi_{s}$. Therefore, in the following we replace

$$
\Psi_{s}(\mathbf{k}) \approx \Psi_{s}\left(k_{F s}, \theta\right),
$$

where $k_{F s}$ is the Fermi wave vector for the spin direction $s$.

Now we expand the function $\Psi$ in harmonics of the angle $\theta$ as in Eq. (17). Inserting Eq. (15) and (17) into the Boltzmann equation gives, for the left-hand side,

$$
\begin{aligned}
\frac{\hbar k_{s x}}{m}( & \left.-\frac{\partial f_{0}\left(\varepsilon_{k s}\right)}{\partial \varepsilon_{k s}}\right)\left(\frac{\partial \Psi_{s}}{\partial x}-e E_{x}\right) \\
= & \sum_{n} \frac{\hbar k_{s} \cos \theta}{m}\left(-\frac{\partial f_{0}\left(\varepsilon_{k s}\right)}{\partial \varepsilon_{k s}}\right)\left(\cos n \theta \frac{\partial g_{s}^{(n)}}{\partial x}-\frac{e E}{\hbar}\right),
\end{aligned}
$$

and for the right-hand side we have two terms. The first one is the spin conserving impurity scattering term, which becomes

$$
H_{0}\left[f_{s}\right]=-\sum_{n} \cos (n \theta) g_{s}^{(n)} \frac{1}{\tau_{\mathrm{tr}}^{n}}\left(-\frac{\partial f_{0}\left(\varepsilon_{k s}\right)}{\partial \varepsilon_{k s}}\right),
$$

where we defined transport times of order $n$,

$$
\frac{1}{\tau_{\mathrm{tr}, s}^{n}}=\int \frac{d \mathbf{k}^{\prime}}{(2 \pi)^{d}} W_{s}^{0}\left(\mathbf{k}, \mathbf{k}^{\prime}\right)\left[1-\cos n \theta_{\mathbf{k}, \mathbf{k}^{\prime}}\right] \delta\left(\varepsilon_{k s}-\varepsilon_{k^{\prime} s}\right),
$$


and where $\theta_{\mathbf{k}, \mathbf{k}^{\prime}}$ is the angle between $\mathbf{k}$ and $\mathbf{k}^{\prime}$. The second term is the one with the $e-e$ scattering. When expansion (17) is inserted into the $e-e$ interaction terms, different $n$ 's do not couple; see, for example, the derivation in Ref. 17. The trick is to write, for example, the angle of $\mathbf{k}^{\prime}-\mathbf{q}$ as $\cos n \theta_{\mathbf{k}^{\prime}-\mathbf{q}, \mathbf{x}}=\cos n\left(\theta_{\mathbf{k}^{\prime}-\mathbf{q}, \mathbf{k}}+\theta_{\mathbf{k}, \mathbf{x}}\right)=\cos n \theta_{\mathbf{k}^{\prime}-\mathbf{q}, \mathbf{k}} \cos n \theta_{\mathbf{k}, \mathbf{x}}$ $-\sin n \theta_{\mathbf{k}^{\prime}-\mathbf{q}, \mathbf{k}} \sin n \theta_{\mathbf{k}, \mathbf{x}}$, and note that the sin terms vanish due to symmetry. Therefore we can express the $e-e$ collision term as

$$
H_{e-e}\left[f_{s}, f_{s^{\prime}}\right](k, \theta)=\sum_{n} \cos (n \theta)\left(g_{s}^{(n)} J^{(n)}+g_{s^{\prime}}^{(n)} I^{(n)}\right),
$$

where $J^{(n)}$ corresponds to the first and third terms in Eq. (18), while $I^{(n)}$ corresponds to the second and fourth terms.

Now a set of equations for the coefficients $g_{s}^{(n)}$ can be extracted by multiplying the Boltzmann equation by $\cos n^{\prime} \theta$ and integrating over $\theta$, while using that $\int d \theta \cos n \theta \cos n^{\prime} \theta$ $\propto \delta_{n n^{\prime}}$. The left-hand side of Eq. (22) is expanded in harmonics, using that $\cos \theta \cos n \theta=\frac{1}{2}[\cos \theta(n+1)+\cos \theta(n-1)]$. We find the following set of equations:

$$
\begin{gathered}
\frac{\partial g_{s}^{(1)}}{\partial x}=0 \\
\frac{\hbar k}{m} \frac{\partial}{\partial x}\left(g_{s}^{(0)}+\frac{g_{s}^{(2)}}{2}+e \phi\right) \eta_{s}=\left(g_{s}^{(1)} \frac{\eta_{s}}{\tau_{\mathrm{tr}}^{1}}+g_{s}^{(1)}\left(J^{(1)}+I^{(1)}\right)\right. \\
\left.+g_{-s}^{(1)} I^{(1)}\right) \\
\frac{1}{2} \frac{\hbar k}{m} \frac{\partial}{\partial x}\left(g_{s}^{(n-1)}+g_{s}^{(n+1)}\right) \eta_{s} \\
=\left(g_{s}^{(n)} \frac{\eta_{s}}{\tau_{\mathrm{tr}}^{n}}+g_{s}^{(n)}\left(J^{(n)}+I^{(n)}\right)+g_{-s}^{(n)} I^{(n)}\right), \quad n \geqslant 2
\end{gathered}
$$

where

$$
\eta_{s}=\left(-\frac{\partial f_{0}\left(\varepsilon_{k s}\right)}{\partial \varepsilon_{k s}}\right) .
$$

The solution of these equations is $g_{s}^{(n)}=0$ for $n \geqslant 2$. This is due to the fact that $\partial_{x} g_{s}^{(1)}=0$, which decouples the equations for $n \geqslant 2$ from the first two equations. Equation (26a) expresses current conservation within each spin species. If we include spin-flip scattering in the equation, then the equations couple because $\partial_{x} g_{s}^{(1)} \neq 0$.

Now we note that setting $g_{s}^{(n)}=0$ for $n \geqslant 2$ corresponds precisely to a linearized shifted Fermi-Dirac distribution

$$
f_{s}^{\mathrm{SFD}}(k)=f_{0}\left(\varepsilon_{s}\left(\mathbf{k}+\delta \mathbf{k}_{s}\right)-\delta \mu_{s}\right),
$$

from which we read off (for $\delta \mathbf{k}$ in the $x$ direction)

$$
g_{s}^{(0)}=\delta \mu_{s}, \quad g_{s}^{(1)} \cos \theta=\hbar v_{x} \delta k_{s} \Rightarrow g_{s}^{(1)}=-\frac{\hbar^{2} k_{F s}}{m} \delta k_{s} .
$$

From Eqs. (26), one can now determine $g_{s}^{0}$ and $g_{s}^{1}$. They correspond to the change of the local charge densities and to the local currents, respectively. We will see this in Sec. IV, where we use the SFD ansatz to study the general case.

\section{MACROSCOPIC TRANSPORT EQUATIONS}

Above we saw that at low temperature the exact solution of the Boltzmann equation in the absence of spin-flip was a shifted Fermi-Dirac function. The same conclusion applies to the situation of arbitrary temperatures but weak $e-e$ scattering, because this limit corresponds to the usual Coulomb drag regime. However, this is no longer necessarily true at arbitrary $e-e$ scattering, when the temperature increases, or when spin-flip processes are included. Nevertheless, we shall assume in the following that the SFD distribution is a good approximation for the exact distribution function. The argument for doing this is as follows: the $e-e$ interactions will drag the distribution functions toward shifted Fermi-Dirac distributions, because the interspin channel $e-e$ collision terms vanish for $f_{s}=f_{s}^{\mathrm{SFD}}$, i.e., $H_{e-e}\left[f_{s}^{\mathrm{SFD}}, f_{s}^{\mathrm{SFD}}\right]=0$. Since the $e$-e scattering rate increases as $\tau_{e-e}^{-1} \approx\left(\varepsilon_{F} / \hbar\right)\left(k T / \varepsilon_{F}\right)^{2}$, increasing the temperature actually helps. Furthermore, since the energy dependence of the elastic scattering is important in determining the actual shape of the distribution functions, and because we do not go into details of this sort, we view the SFD distribution functions as reasonable parametrizations of the true distribution function.

Our starting point is thus an ansatz distribution function given by

$$
\begin{aligned}
f_{s}(k)= & f_{0}\left(\varepsilon_{k s}\right)+\left(-\frac{\partial f_{0}\left(\varepsilon_{k s}\right)}{\partial \varepsilon_{k s}}\right) \delta \mu_{s}(x) \\
& -\left(-\frac{\partial f_{0}\left(\varepsilon_{k s}\right)}{\partial \varepsilon_{k s}}\right) \hbar v_{x} \delta k_{s}(x) .
\end{aligned}
$$

Here $\delta \mu_{s}$ corresponds to a change of the local chemical potential, and hence also to the local density, while $\delta k_{s}$ describes a shift of the distribution function in $\mathbf{k}$ space and thus gives a finite drift velocity. Inserting this into the Boltzmann equation gives, for the left-hand side,

$$
\mathcal{L}=v_{x}\left(-\frac{\partial f_{0}\left(\varepsilon_{k s}\right)}{\partial \varepsilon_{k s}}\right)\left(\frac{\partial}{\partial x}\left(\delta \mu_{s}-\hbar v_{x} \delta k_{s}\right)-e E_{x}\right),
$$

and for the right-hand side we have three terms. The spinconserving collision term becomes

$$
H_{0}\left[f_{s}\right]=-\hbar v_{x} \delta k_{s} \frac{1}{\tau_{\mathrm{tr}, s}}\left(-\frac{\partial f_{0}\left(\varepsilon_{k s}\right)}{\partial \varepsilon_{k s}}\right),
$$

where the usual transport time is 


$$
\frac{1}{\tau_{\mathrm{tr}, s}}=\int \frac{d \mathbf{k}^{\prime}}{(2 \pi)^{d}} W_{s}^{0}\left(\mathbf{k}, \mathbf{k}^{\prime}\right)\left[1-\cos \theta_{\mathbf{k}, \mathbf{k}^{\prime}}\right] \delta\left(\varepsilon_{k s}-\varepsilon_{k^{\prime} s}\right)
$$

The second term on the right-hand side is the spin-flip scattering term, which becomes

$$
\begin{aligned}
H_{\mathrm{sf}}\left[f_{s}, f_{-s}\right]= & \left(\hbar v_{x} \delta k_{s} \frac{1}{\tau_{\mathrm{sf}, \mathrm{tr}}}-\left(\delta \mu_{s}-\delta \mu_{-s}\right) \frac{1}{\tau_{\mathrm{sf}}^{0}}-\hbar v_{x}\right. \\
& \left.\times\left(\delta k_{s}-\delta k_{-s}\right) \frac{1}{\tau_{\mathrm{sf}}}\right)\left(-\frac{\partial f_{0}\left(\varepsilon_{k s}\right)}{\partial \varepsilon_{k s}}\right),
\end{aligned}
$$

where the three different spin-flip scattering times are given by

$$
\begin{gathered}
\frac{1}{\tau_{\mathrm{sf}}^{0}}=\int \frac{d \mathbf{k}^{\prime}}{(2 \pi)^{d}} W_{s f}\left(\mathbf{k}, \mathbf{k}^{\prime}\right) \delta\left(\varepsilon_{k s}-\varepsilon_{k^{\prime}-s}\right), \\
\frac{1}{\tau_{\mathrm{sf}, \mathrm{tr}}}=\int \frac{d \mathbf{k}^{\prime}}{(2 \pi)^{d}} W_{s f}\left(\mathbf{k}, \mathbf{k}^{\prime}\right)\left[1-\cos \theta_{\mathbf{k}, \mathbf{k}^{\prime}}\right] \delta\left(\varepsilon_{k s}-\varepsilon_{k^{\prime}-s}\right), \\
\frac{1}{\tau_{\mathrm{sf}}}=\int \frac{d \mathbf{k}^{\prime}}{(2 \pi)^{d}} W_{s f}\left(\mathbf{k}, \mathbf{k}^{\prime}\right) \cos \theta_{\mathbf{k}, \mathbf{k}^{\prime}} \delta\left(\varepsilon_{k s}-\varepsilon_{k^{\prime}-s}\right) .
\end{gathered}
$$

Finally, the $e$-e scattering is given by $\Sigma_{s^{\prime}} H_{e-e}\left[f_{s}, f_{s^{\prime}}\right]$. But, in accordance with detailed balance, the $e-e$ scattering between two identical Fermi-Dirac distributions is zero, $H_{e-e}\left[f_{s}, f_{s}\right]=0$, and we are left with

$$
\begin{aligned}
H_{e-e}\left[f_{s}, f_{-s}\right]= & -\frac{\hbar^{2}}{m} \int_{-\infty}^{\infty} \frac{d \omega}{2 \pi} \int \frac{d \mathbf{k}^{\prime}}{(2 \pi)^{d}} \int \frac{d \mathbf{q}}{(2 \pi)^{d}} \\
& \times \mid U_{s,-s}\left(\mathbf{q}, \varepsilon_{k}-\left.\varepsilon_{\mid \mathbf{k}+\mathbf{q})}\right|^{2}\right. \\
& \times \frac{1}{k T \sinh ^{2}\left(\hbar \omega / k_{B} T\right)} \operatorname{Im} \chi_{s}(\mathbf{k}, \mathbf{q} ; \omega) \\
& \times \operatorname{Im} \chi_{-s}\left(\mathbf{k}^{\prime},-\mathbf{q} ; \omega\right) q_{x}\left[\delta k_{s}-\delta k_{-s}\right] .
\end{aligned}
$$

The final form of the Boltzmann equation is thus

$$
(31)=(32)+(34)+(36) .
$$
by

Next we find the current and the density. They are given

$$
\begin{aligned}
J_{s} & \equiv-e \int \frac{d \mathbf{k}}{(2 \pi)^{d}} v_{x} f_{s}(\mathbf{k}) \\
& =e \int \frac{d \mathbf{k}}{(2 \pi)^{d}} \hbar v_{x}^{2}\left(-\frac{\partial f_{0}\left(\varepsilon_{k s}\right)}{\partial \varepsilon_{k s}}\right) \delta k_{s}=\frac{\hbar e}{m} n_{s}^{0} \delta k_{s},
\end{aligned}
$$

$$
\begin{aligned}
\delta \rho_{s} & \equiv-e \int \frac{d \mathbf{k}}{(2 \pi)^{d}}\left[f_{s}(\mathbf{k})-f_{s}^{0}(\mathbf{k})\right] \\
& =-e \int \frac{d \mathbf{k}}{(2 \pi)^{d}}\left(-\frac{\partial f_{0}\left(\varepsilon_{k s}\right)}{\partial \varepsilon_{k s}}\right) \delta \mu_{s}=-e \frac{\partial n_{s}^{0}}{\partial \mu} \delta \mu_{s} .
\end{aligned}
$$

We find two transport equations for the current and charge density or chemical potentials by integrating Eq. (37) and also Eq. (37) multiplied by $v_{x}$ with respect to $\mathbf{k}$, and we arrive at Eq. (1), where

$$
\alpha_{s}=\frac{n_{s}^{0}}{n_{-s}^{0}},
$$

$$
\begin{gathered}
\sigma_{s}=\frac{n_{s}^{0} e^{2}}{m} e\left(\frac{1}{\tau_{\mathrm{tr}, s}}+\frac{1}{\tau_{\mathrm{sf}, \mathrm{tr}}}\right)^{-1}, \\
\sigma_{\mathrm{sf}, s}=\frac{n_{s}^{0} e^{2} \tau_{\mathrm{sf}}}{m} .
\end{gathered}
$$

In Eq. (1a) we introduced the drag conductivity defined in Eq. (2). In deriving the drag term, we have made use of the result obtained for Coulomb drag in, e.g., Ref. 13. Furthermore, the local electrochemical potential has been defined as

$$
\bar{\mu}_{s}=\mu_{s}+e \phi
$$

where $\phi$ is the electrical potential.

\section{EVALUATION OF THE SPIN DRAG RESISTIVITY}

\section{A. One dimension}

The polarization function is in one dimension at small temperatures, where we can perform an $\omega$ expansion, given by

$$
\operatorname{Im} \chi_{s}(q, \omega) \approx \omega \frac{m^{2}}{4 \hbar^{3} q^{2}}\left(-\frac{\partial f_{0}\left(\varepsilon_{q / 2, s}\right)}{\partial \varepsilon_{q / 2, s}}\right) .
$$

Inserting this into the formula for $\sigma_{D}^{-1}$, performing the $\omega$ integration for the case of a nonmagnetic conductor $\varepsilon_{k s}$ $=\varepsilon_{k}$, and using that $\left[f_{0}^{\prime}(\varepsilon)\right]^{2} \simeq(6 k T)^{-1} \delta\left(\varepsilon-\varepsilon_{F}\right)$, we find

$$
\sigma_{D}^{-1} \approx \frac{k T}{\varepsilon_{F}} \frac{\pi^{2} k_{F}^{3}}{64} \frac{\hbar}{e^{2}} \frac{\left|U\left(2 k_{F}\right)\right|^{2}}{\varepsilon_{F}^{2}} .
$$

The spin drag resistance is thus proportional to temperature and dependent on the Coulomb backscattering matrix element. Clearly, this contribution can be very large at finite temperatures. However, in strictly one dimensions, where Fermi-liquid theory is not expected to apply, the Boltzmann equation is not a correct starting point, and one should be somewhat careful about drawing firm conclusions from this. Nevertheless, this Fermi golden rule result is indicative of $e-e$ interactions being very important for spin transport in one dimension. 


\section{B. Two dimensions}

For the two-dimensional case we start by deriving a lowtemperature result, and go on to compare it with a full numerical integration of $\sigma_{D}^{-1}$. At small $\omega$ and $q$ the imaginary part of the polarization function is given by

$$
\operatorname{Im} \chi_{s}(q, \omega) \approx \omega \frac{m^{2}}{2 \pi \hbar^{3} q^{3} k_{F}},
$$

and the screened static Coulomb interaction is

$$
U(q)=\frac{e^{2}}{2 \pi \varepsilon_{0} \varepsilon_{r}} \frac{1}{\left(q+q_{T F}\right)} .
$$

In this approximation, the $\mathbf{q}$ integral becomes

$$
\int d q q^{3} \frac{1}{\left(q+q_{T F}\right)^{2}},
$$

which is clearly not convergent, and therefore we set the upper limit to be $2 k_{F}$, because $\operatorname{Im} \chi$ is zero for a momentum exchange larger than $2 k_{F}$. With these inputs, we arrive at the approximate expression

$$
\sigma_{D}^{-1} \approx\left(\frac{k T}{\varepsilon_{F}}\right)^{2} \frac{\hbar}{e^{2}} \frac{\pi^{2}}{3} \frac{(1+\gamma) \ln (1+\gamma)-\gamma}{\gamma^{2}(1+\gamma)},
$$

where $\gamma=2 k_{F} / q_{T F}$ and $q_{T F}=m e^{2} / 2 \pi \varepsilon_{0} \varepsilon_{r} \hbar^{2}$ is the twodimensional inverse Thomas-Fermi screening length. Typically $\gamma$ is of order 1 . This means that the spin drag resistivity can be equal to a fraction of the quantum resistance, and should therefore indeed be measurable for standard highmobility quantum wells.

We have also integrated the spin drag formula numerically; see Ref. 14 for details. The result is shown in Fig. 1 for realistic numbers for a two-dimensional GaAs electron gas. The integration is done using the full dynamically screened interaction for a quantum well with finite thickness. The approximate formula [Eq. (46)] is seen to overestimate the spin drag effect slightly.

\section{CONCLUSIONS}

We have derived a set of transport equations for spinpolarized drag which incorporate $e-e$ scattering. This has been done within the framework of the Boltzmann equation. First we showed that in the absence of spin-flip scattering and at low temperatures the exact solution of the Boltzmann

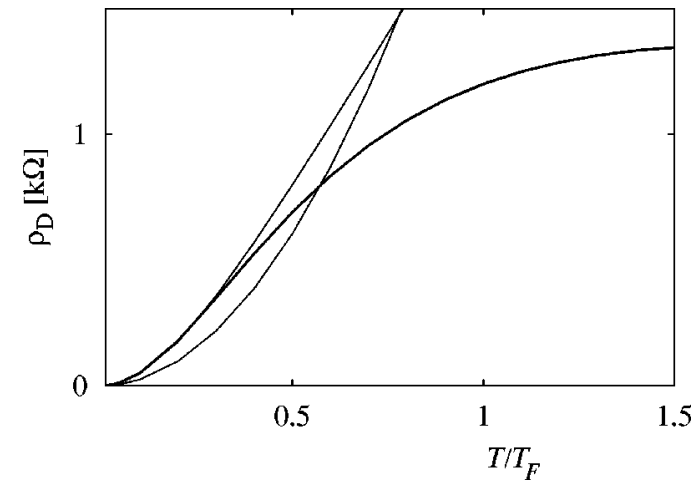

FIG. 1. The spin drag resistivity in the two-dimensional case as a function of temperature. The thick line is the numerical integration of Eq. (2) for a two-dimensional quantum well of thickness 10 $\mathrm{nm}$ and electron density $2 \times 10^{15} \mathrm{~m}^{-2}$. We have used typical parameters for GasAs-based heterostructures. The rightmost thin line is the approximate expression in Eq. (46), while the left thin line is the result of integrating Eq. (2), but using the $T=0$ expression for $\chi(q, \omega)$.

equation corresponds to two shifted Fermi-Dirac distribution functions. Furthermore, if the interaction is weak, one can use perturbation theory and arrive at the same conclusion following the lines of argument from Coulomb drag. Having observed that the shifted Fermi-Dirac distribution is correct at low temperatures or weak $e-e$ scattering, we go on to the general case, which is solved approximately by using the SFD as an ansatz, which allows for a solution of the coupled Boltzmann equations.

The main conclusion from this is that $e-e$ interaction introduces a spin drag term, which tend to drag the spin currents to be equal. There are two such mechanisms, namely, $e-e$ interactions, which is temperature dependent, and angular-dependent elastic spin-flip scattering, which is temperature independent. Therefore, if a spin-polarized current is driven through the system, the spin drag will give rise to an additional resistivity. This resistivity increases with temperature. We have solved for the spin drag resistivity numerically in two dimensions, which shows that it can become considerable and even exceed the ordinary impurityscattering-induced resistivity. The spin drag should thus be measurable in, for example, a structure combining a twodimensional electron gas with ferromagnetic materials or for one-dimensional systems, e.g., fabricated by nanotechnology in semiconductors or by contacting nanotubes to ferromagnetic contacts.
${ }^{1}$ P. M. Levy, Solid State Phys. 47, 367 (1994).

${ }^{2}$ See, e.g., in Proceedings of First International Conference of the Physics and Applications of Spin-related Phenomena in Semiconductors, edited by Katayama [Physica E 10 (2001)].

${ }^{3}$ M. Johnson and R. H. Silsbee, Phys. Rev. B 35, 4959 (1987).

${ }^{4}$ P. C. van Son, H. van Kempen, and P. Wyder, Phys. Rev. Lett. 58, 2271 (1987).
${ }^{5}$ T. Valet and A. Fert, Phys. Rev. B 48, 7099 (1993).

${ }^{6}$ G. Schmidt, D. Ferrard, L. W. Molenkamp, A. T. filip, and B. J. van Wees, Phys. Rev. B 62, R4790 (2000).

${ }^{7}$ I. Zutik, J. Fabian, and S. Das Sarma, cond-mat/0106085.

${ }^{8}$ I. D'Amico and G. Vignale, Phys. Rev. B 62, 4853 (2000).

${ }^{9}$ I. D'Amico and G. Vignale, Europhys. Lett. 55, 566 (2001).

${ }^{10}$ Y. Takahashi, K. Shizume, and N. Masuhara, Phys. Rev. B 60, 
4856 (1999).

${ }^{11}$ Y. Takahashi, K. Shizume, and N. Masuhara, Physica E 10, 22 (2001)

${ }^{12}$ W. J. Mullin and J. W. Leon, J. Low Temp. Phys. 88, 433 (1992).

${ }^{13}$ A.-P. Jauho and H. Smith, Phys. Rev. B 47, 4420 (1993).

${ }^{14}$ K. Flensberg and B. Y.-K. Hu, Phys. Rev. B 52, 14796
(1995).

${ }^{15}$ B. Y.-K. Hu and K. Flensberg, in Hot Carriers in Semiconductors, edited by K. Hess (Plenum Press, New York, 1996), p. 943.

${ }^{16} \mathrm{H}$. Smith and H. H. Jensen, Transport Phenomena (Clarendon Press, Oxford, 1989).

${ }^{17}$ B. Y.-K. Hu and K. Flensberg, Phys. Rev. B 53, 10072 (1996). 